\title{
Implementation of PNN Based on Extraction of DCT features for Brain Tumor Classification
}

\author{
Priyanka Katti ${ }^{1}$, Mr. V. R. Marathe ${ }^{2}$ \\ Student, E \& TC, N. B. Navale Sinhgad College of Engineering, Solapur, Maharashtra, India ${ }^{1}$ \\ Assistant Professor, E \& TC, N. B. Navale Sinhgad College of Engineering, Solapur, Maharashtra, India ${ }^{2}$
}

\begin{abstract}
Accurate detection, segmentation and classification of Brain tumor is a challenging task in medical field. This paper proposes a method for brain tumor classification using Probabilistic Neural Network based on textural feature extraction of Magnetic Resonance Images (MRI). The proposed scheme consist of several stages including image acquisition, segmentation of tumor part, feature extraction and classification. This project include clustering algorithm for segmentation of MRI to detect brain tumor. Discrete cosine transform (DCT) is used for the dimensionality reduction and feature extraction. PNN will be useful to classify the stages of brain tumor that is Normal, Benign or Malignant. This can be performed in two stages : 1) Gray level co-occurrence matrix and 2) Classification using PNN based function. Detection and extraction of tumor from MRI scan images of the brain is done by using MATLAB software. Evaluation was performed on image database of 90 MRI of brain.
\end{abstract}

Keywords: Discrete Cosine Transform(DCT), Gray level co-occurrence matrix(GLCM), MATLAB, K-means Clustering, Magnetic Resonance Images(MRI), Probabilistic Neural Network.

\section{INTRODUCTION}

Brain tumors are caused by an abnormal \& uncontrolled growth of cells or cell division. Brain tumors may also spread from cancers primarily located in the other organs also known as secondary brain tumors. Tumor is mass of tissues that grows out of control. It interfaces with normal functioning of brain. Tumor shifts the brain towards skull $\&$ increases the pressure on the brain. Detection of tumor is first step in treatment. The MRI brain image is acquired from patient's database, internet and then Image acquisition, image segmentation is performed for brain tumor detection. Because of many types brain tumors, classification becomes very complicated. So classification of brain tumor is very important part. A good classification process leads to the right decision and provide proper treatment.

Brain tumors are classified based on the type of tissue involved, size of tumor, the location of the tumor, whether it is normal, benign or malignant.

Normal Brain: If there is not any unwanted growth of cells then it is non cancerous brain. It does not consist cancer cell. So it is Normal brain[4].

Benign brain tumor: This type of tumor generally do not consist cancer cells and can be removed. Benign brain tumors usually have an obvious border or edge[4]. They don't spread to other parts of the body. However, benign tumors can cause serious health problems.

Malignant brain tumor: This consists of cancer cells and hence also called as brain cancer. They are likely to grow rapidly and can affect nearby healthy brain tissues. This type of tumor can be a threat for life[4]. Malignant brain tumors do not have an specific border or edge.

\section{PROPOSED METHODOLOGY}

The concept of Probabilistic Neural Network is used for the effective and accurate classification. Decision making was performed in two stages: feature extraction using Discrete Cosine transform (DCT) and the Probabilistic Neural Network (PNN)[1]. This project classifies a brain tumor if it is a benign tumor, a malignant tumor or normal one. Probabilistic Neural Network gives fast and accurate classification and is a promising tool for classification of the tumors.

\section{A. Image acquisition :}

Maximum MRI Images of brain are collected form possible resources like Radiologists, Internet, and Hospitals etc. Images are obtained using MRI scan \& displayed in 2D having pixels as its elements. MRI scan were stored in database of images in JPEG image formats. These images are displayed as a gray scale images. The entries of gray scale images are ranging from 0 to 255 , where o indicates total black color and 255 represents total white colour.

\section{B. Segmentation using Clustering:}

In this paper segmentation is performed by using clustering method and morphological operations.

1) K-means clustering \& its algorithm :

$K$ clusters $\mathrm{K}$-means is one of the simplest unsupervised learning algorithms that solve the well known clustering problem by making partitions in an image. Assume $\mathrm{k}$ number of clusters to classify the database by defining $\mathrm{k}$ centroids one for each cluster. The next step is to take each point belonging to a given data set and associate it to the nearest centroid[2].

Algorithm is as follows :

Step1. Choose the number K of clusters either manually, randomly or based on some heuristic. 
Step2. Generate K clusters and determine the cluster's center.

Step3. Assign each pixel in the image to the cluster that minimizes the variance between the pixel and the cluster center

Step4. Re-compute cluster centers by averaging all of the pixels in the cluster.

Step5. Repeat steps 3 and 4 until some convergence criterion is met[2].

\section{2) Morphological operations:}

In this paper morphological operators are used for the tumor region extraction and to further remove the non tumor regions[6]. In tumor regions, vertical edges, horizontal edges, and diagonal edges are mingled together while they are distributed separately in non tumor regions[6]. The dilation operator is used for filling the broken gaps at the edges and to have continuities at the boundaries.

\section{Feature Extraction :}

The extraction of texture features in the detected tumor has been achieved by using Gray Level Co-occurrence Matrix (GLCM)[4][3]. The method calculates a GLCM by calculating how often a pixel with a certain intensity $i$ occurs in relation with another pixel $\mathrm{j}$ at a certain distance $\mathrm{d}$ and orientation $\theta$. Co-occurrence matrices are calculated for four directions: $0^{\circ}, 45^{\circ}, 90^{\circ}$ and $135^{\circ}$ degrees. The eight Haralick texture descriptors are extracted from each co-occurrence matrices which are computed in each of four angles.

Five features extracted in this paper are explained below.

(i) Contrast:

Contrast is defined as the separation between the darkest and brightest area.

$$
\text { Contrast }=\sum_{i, j=0}^{n-1} P_{i, j}(i-j)^{2}
$$

(ii)Correlation:

Correlation is computed into what is known as the correlation coefficient, which ranges between -1 and +1 .

$$
\text { Correlation }=\sum_{i, j=0}^{n-1} P_{i j} \frac{(i-\mu)(j-\mu)}{\sigma^{2}}
$$

(iii)Homogeneity:

Homogeneity is defined as the quality or state of being homogeneous.

$$
\text { Homogenity }=\sum_{i, j=0}^{n-1} \frac{P_{i j}}{1+(i-j)^{2}}
$$

(iv)Entropy :

Entropy is a measure of the uncertainty in a random variable.

$$
\text { Entropy }=\sum_{i, j=0}^{N-1}-\ln \left(P_{i j}\right) P_{i j}
$$

(v)Energy :

It provides the sum of squared elements in the GLCM .Also known as the uniformity or the angular second moment.

$$
\text { Energy }=\sum_{i, j=0}^{N-1}\left(P_{i j}\right)^{2}
$$

\section{Discrete Cosine Transform :}

In the proposed technique DCT features of an images are extracted as an initial step. On the basis of these features brain MR images are classified as normal or abnormal. DCT operates on a function at finite number of discrete data points[1]. The DCT has the property that, for a typical image, most of the visually significant information is concentrated in just a few coefficients[1].

The DCT of an M x N gray scale matrix of the image $f(x$, $\mathrm{y})$ is defined as follows:

$$
\begin{aligned}
& T(u, v)=\sum_{x=0}^{M-1} \sum_{y=0}^{N-1} f(x, y) \alpha(u) \alpha(v) \times \\
& \cos \left[\frac{(2 x+1) u \pi}{2 M}\right] \cos \left[\frac{(2 y+1) v \pi}{2 N}\right] \\
& \text { Where } \begin{aligned}
\alpha(u) & =\sqrt{\frac{1}{M}} \text { for } \mathrm{u}=0 \\
& =\sqrt{\frac{2}{M}} \text { for } \quad \mathrm{u}=1,2, \ldots, \mathrm{M}-1
\end{aligned}
\end{aligned}
$$

The values $\mathrm{T}(\mathrm{u}, \mathrm{v})$ are the DCT coefficients.

\section{E. Training and Testing:}

In the training phase, feature vectors are extracted for each image in the training set. Features are extracted through GLCM. Set of extracted features for each image is known as feature vector. Feature vectors for every image is computed as $[\mathrm{x} 1 \mathrm{x} 2 \times 3 \ldots \mathrm{xn}]$.

In the testing phase, the feature vector [y1 y2 y3..yn] of the test image is computed using DCT. In order to identify the test image, the similarities between test image and all of the feature vectors in the training set are computed. The similarity between feature vectors is computed using Euclidean distance. The identity of the most similar image is the output of the image recognizer. If feature vector of test image matches with feature vector of image in training set then test image has correctly identified.

\section{F. Probabilistic Neural Network :}

A probabilistic neural network (PNN) is a feed forward neural network, which was derived from the Bayesian network and a statistical algorithm called Kernel Fisher discriminate analysis. It was introduced by D.F. Specht in the early 1990s. In a PNN, the operations are organized into a multilayered feed forward network with four layers:

- Input layer

- Hidden layer

- Pattern layer/Summation layer

- Output layer 
In 1990, Donald F. Specht proposed a method to formulate input neurons feed the values to each of the neurons in the the weighted-neighbour method in the form of a neural hidden layer. network. He called this a "Probabilistic Neural Network". Following is a diagrammatic representation of a PNN network :

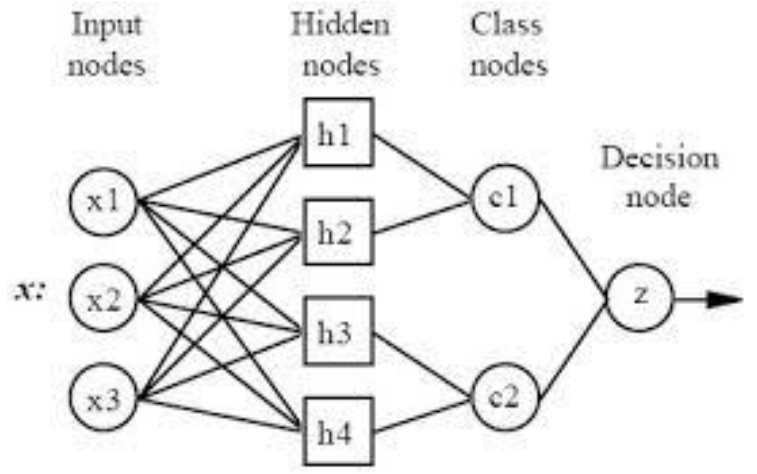

Fig 1. Architecture of Probabilistic Neural Network

PNN is often used in classification problems. When an input is present, the first layer computes the distance from the input vector to the training input vectors[3]. This produces a vector where its elements indicate how close the input is to the training input[4]. The second layer sums the contribution for each class of inputs and produces its net output as a vector of probabilities.

Finally, a complete transfer function on the output of the second layer picks the maximum of these probabilities, and produces a 1 (positive identification) for that class and a 0 (negative identification) for non-targeted classes[4]. Layers of PNN are as shown in the following figure :

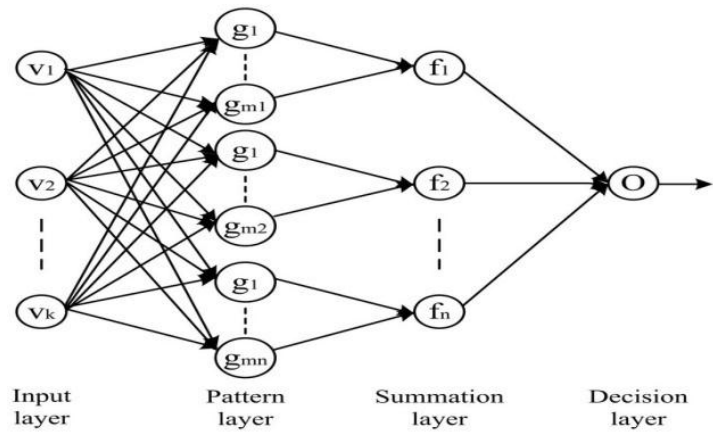

Fig 2. Layers of PNN

Input layer:

Each neuron in the input layer represents a predictor variable. In categorical variables, $N-1$ neurons are used when there are $N$ number of categories. It standardizes the range of the values by subtracting the median and dividing by the interquartile range. Then the

\section{Pattern layer:}

This layer contains one neuron for each case in the training data set. It stores the values of the predictor variables for the case along with the target value. A hidden neuron computes the Euclidean distance of the test case from the neuron's center point and then applies the RBF kernel function using the sigma values.

\section{Summation layer:}

For PNN networks there is one pattern neuron for each category of the target variable. The actual target category of each training case is stored with each hidden neuron; the weighted value coming out of a hidden neuron is fed only to the pattern neuron that corresponds to the hidden neuron's category. The pattern neurons add the values for the class they represent.

\section{Output layer:}

The output layer compares the weighted votes for each target category accumulated in the pattern layer and uses the largest vote to predict the target category.

\section{EXPERIMENTAL RESULTS AND ACCURACY ANALYSIS}

Data base consist of MRI of brain. In this method different images from different patients have been considered, analyzed and classified. Here results for 3 test images are shown out of 7 test images. Following table shows input image, segmentation of tumor and assigned class.

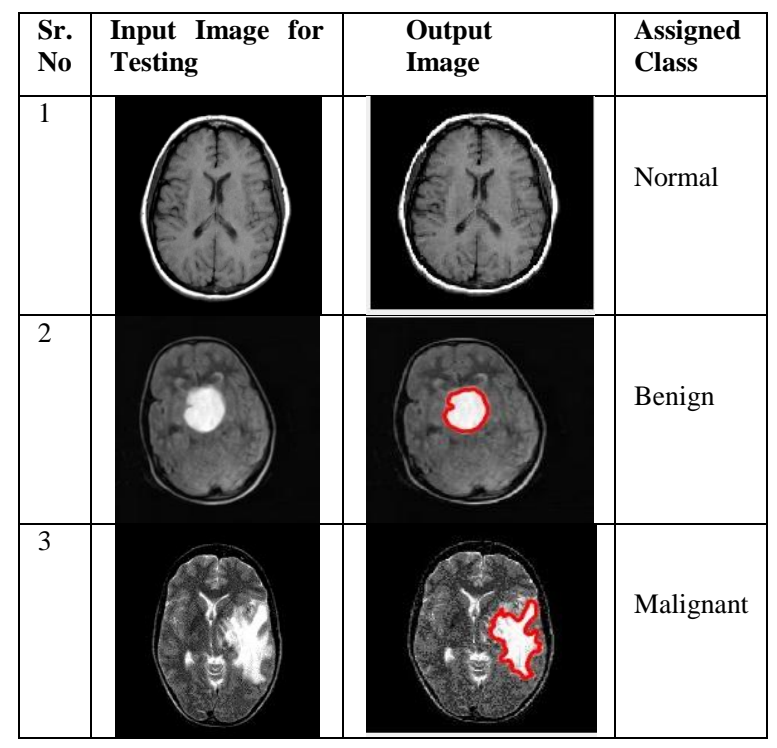

Table 1. Result for classification

A .Accuracy Analysis :

\begin{tabular}{|c|l|c|c|c|}
\hline Sr. No & Test Image & Original Class & Classified Class & Accuracy \\
\hline 1 & Image1 & Normal & Normal & 99.5716 \\
\hline 2 & Image2 & Benign & Benign & 98.4429 \\
\hline 3 & Image3 & Benign & Benign & 98.1795 \\
\hline 4 & Image4 & Benign & Benign & 98.5279 \\
\hline 5 & Image5 & Malignant & Malignant & 99.5292 \\
\hline 6 & Image6 & Malignant & Malignant & 99.4431 \\
\hline 7 & Image7 & Malignant & Malignant & 99.4562 \\
\hline
\end{tabular}

Table 2. Accuracy Analysis for assigned classes 
INTERNATIONAL JOURNAL OF INNOVATIVE RESEARCH IN ELECTRICAL, ELECTRONICS, INSTRUMENTATION AND CONTROL ENGINEERING Vol. 3, Issue 10, October 2015

\section{B. Extracted features :}

\begin{tabular}{|c|c|c|c|c|c|}
\hline Sr. No & Energy & Contrast & Correlation & Homogeneity & Entropy \\
\hline $\mathbf{1}$ & 0.0757 & 921.9987 & 0.2527 & 0.3809 & 7.0493 \\
\hline $\mathbf{2}$ & 0.1350 & 246.8799 & 0.3335 & 0.5617 & 4.9761 \\
\hline $\mathbf{3}$ & 0.1264 & 209.6587 & 0.3881 & 0.5434 & 5.0023 \\
\hline $\mathbf{4}$ & 0.1343 & 261.3241 & 0.4410 & 0.5536 & 5.2635 \\
\hline $\mathbf{5}$ & 0.0858 & 837.8144 & 0.3113 & 0.4075 & 6.9350 \\
\hline $\mathbf{6}$ & 0.0644 & 706.6521 & 0.1481 & 0.3546 & 7.0025 \\
\hline $\mathbf{7}$ & 0.0823 & 724.5247 & 0.2556 & 0.4322 & 6.2865 \\
\hline
\end{tabular}

Table 3. Extracted features for 7 test image

\section{Simulation Results :}

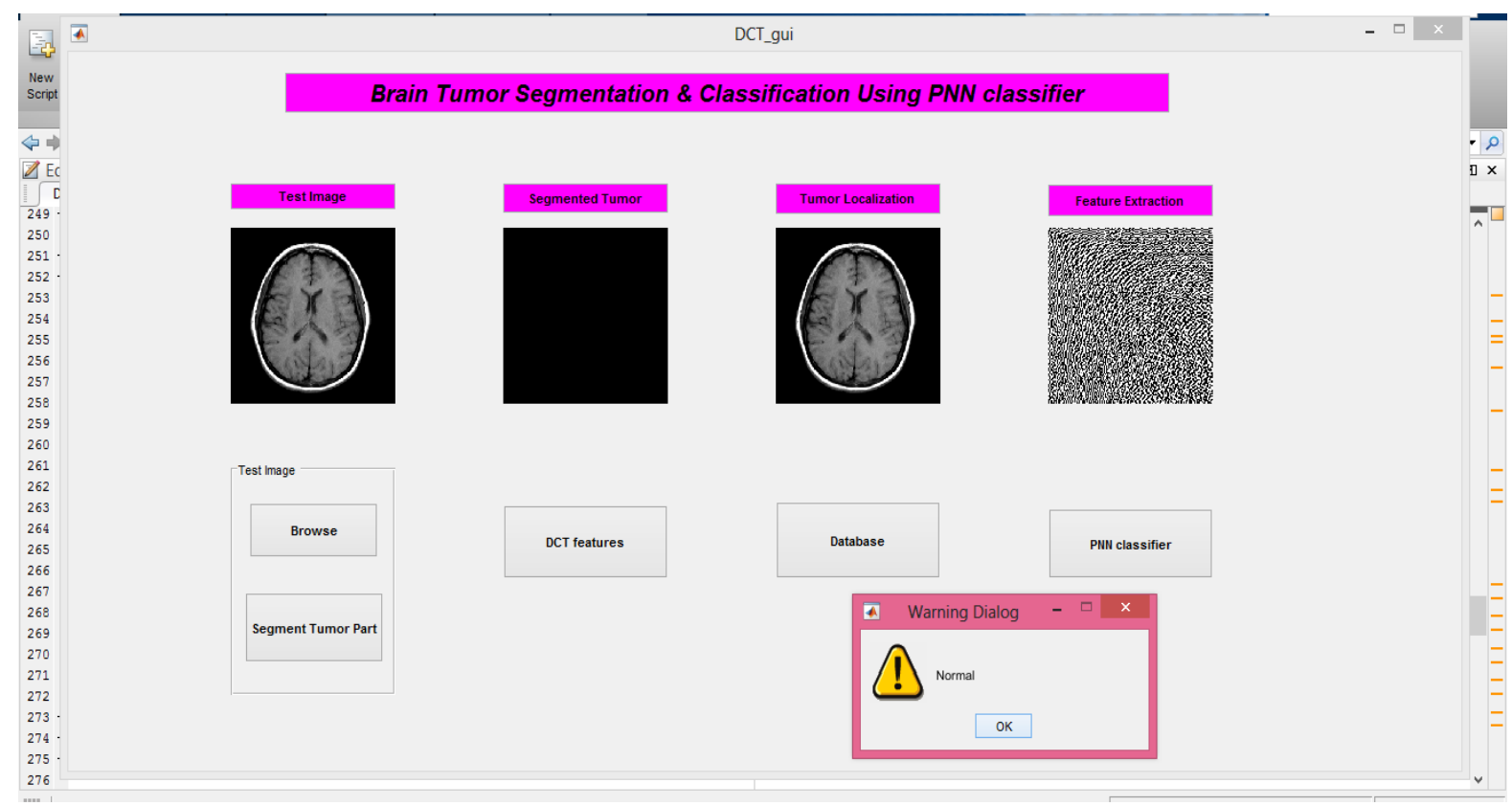

Fig.3 Result for Normal Brain

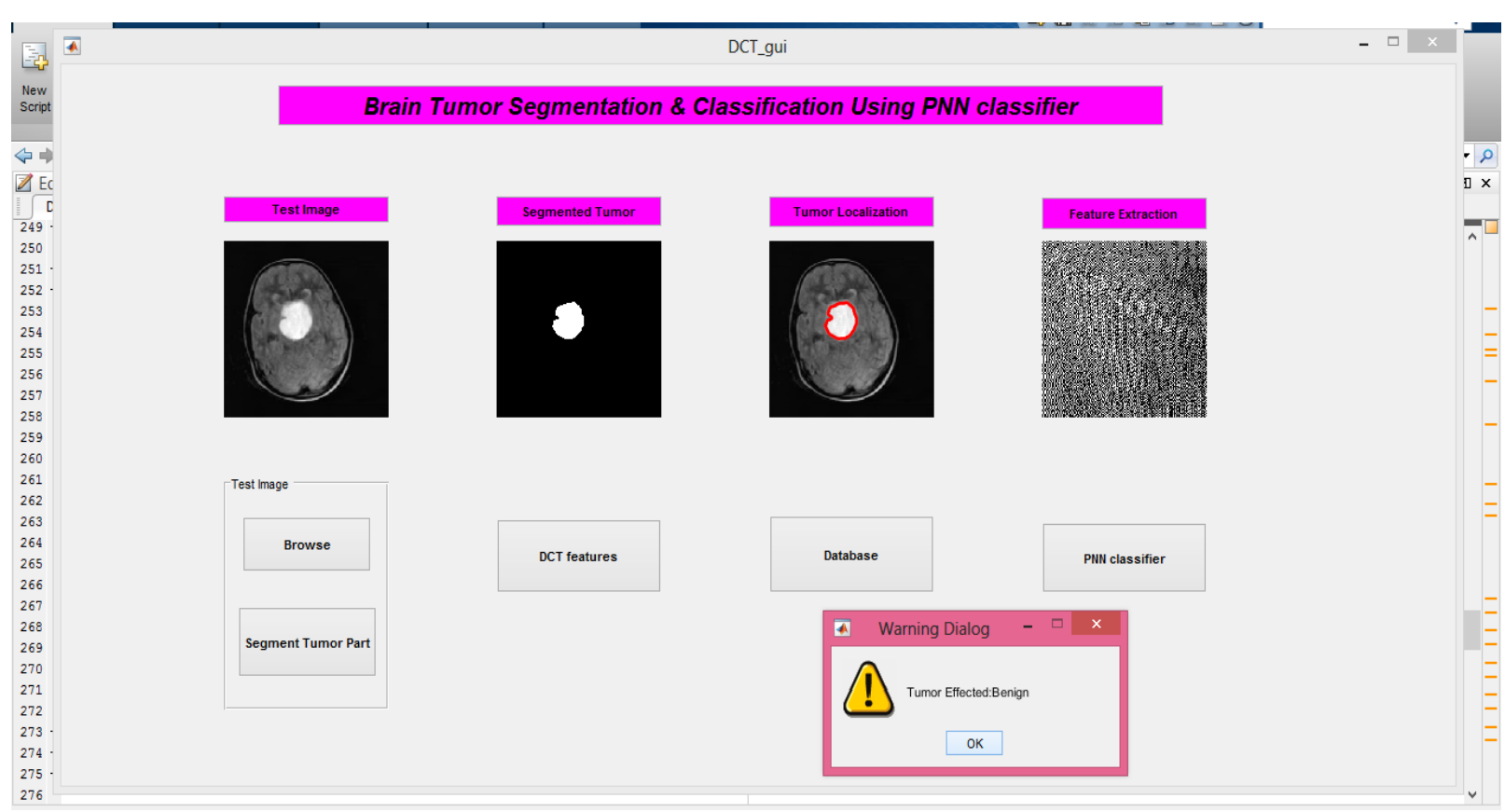

Fig.4 Result for Benign type of Tumor 


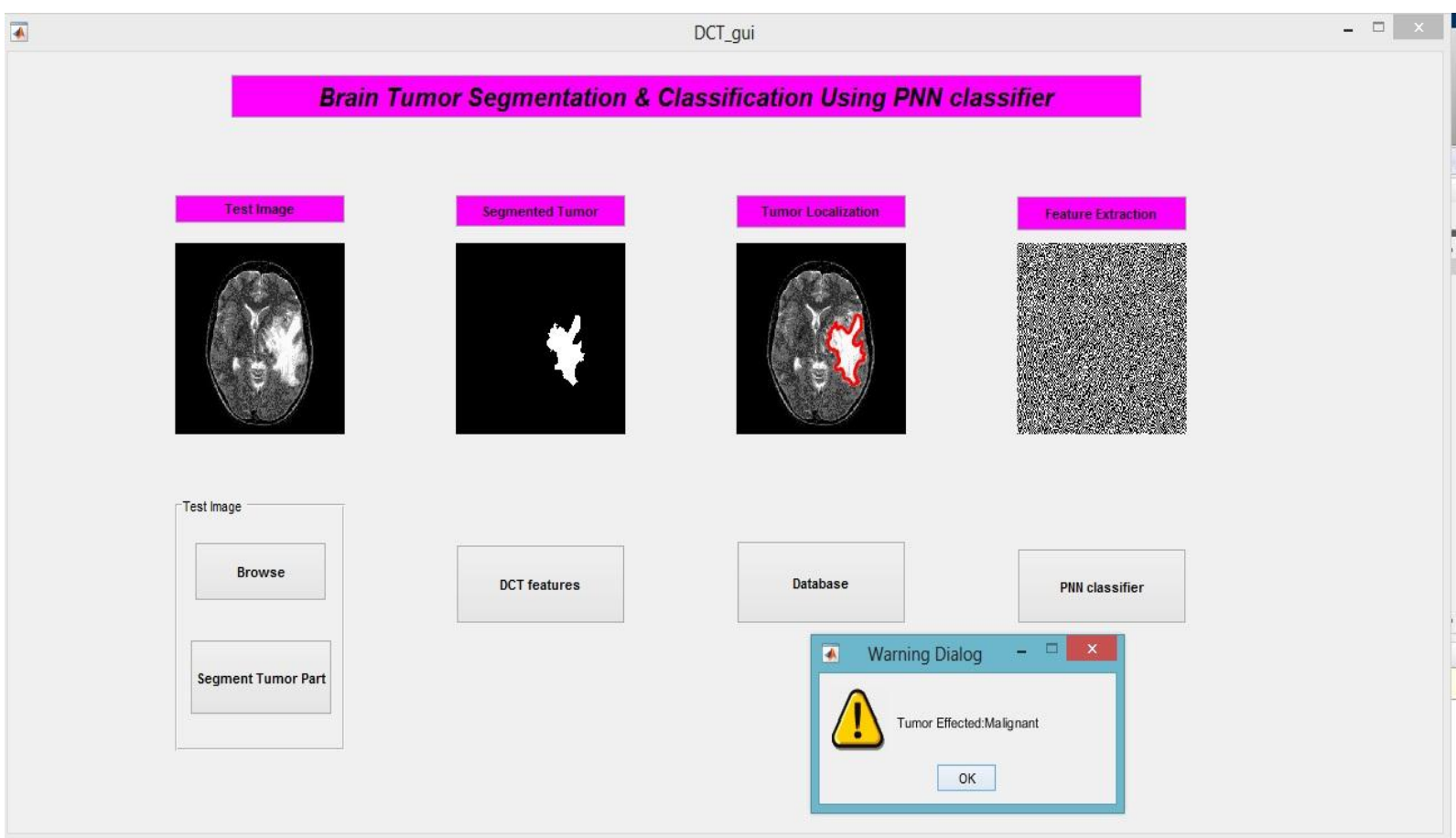

Fig.5 Result for Malignant type of Tumor

\section{CONCLUSION}

Probabilistic Neural Network is used for classification of MRI of brain. It gives accurate results. By using clustering, segmentation is performed accurately. Classification is performed on the basis of DCT feature extraction. On an average this implementation gives $99.99 \%$ of accuracy which will be helpful for diagnosis of brain tumor in early stages.

\section{ACKNOWLEDGMENT}

I would like to thank Prof. V. R. Marathe and all other staffs of E \& TC department of N. B. Navale Sinhgad College of Engineering, Solapur for their valuable support.

\section{REFERENCES}

[1] "Brain Tumor Classification Using Discrete Cosine Transform and Probabilistic Neural Network" D. SRIDHAR, Associate Professor Balaji Institute of Technology and Science, Warangal Andhra pradesh, India.sreedhardsr@yahoo.co.in N. MURALI KRISHNA, Chief Consultant Offshore Programs in Asia,Jackson State University, USA Hyderabad, India. iyyanki@gmail.com 978-14673-4862-1/13/\$31.00 @2013 IEEE

[2] "A Comparative Analysis of Fuzzy C-Means Clustering and K Means Clustering Algorithms" Mrs. Bharati R.Jipkate and Dr.Mrs.V.V.Gohokar SSGMCE, Shegaon, Maharashtra-443101 (India) IJCER | May-June 2012 | Vol. 2 | Issue No.3 |737-739

[3] Classification of Brain Tumor Using Discrete Wavelet Transform, Principal Component Analysis and Probabilistic Neural Network Swapnali Sawakare and Dimple Chaudhari 1Swapnali Sawakare, Electronics and Telecommunication/ Y.T.I.E.T/ Mumbai University, Neha A.P.T, G-1 blk.no. 460/919 A bhd. Lalchakki (Ulhasnagar-4) India 1swapnali.sawakare@gmail.com Dimple Chaudhari, Electronics and Telecommunication/ Y.T.I.E.T/ Mumbai University,India 2dimple.chaudhari@tasgaonkartech.com VOLUME-1, ISSUE-6, NOVEMBER-2014

[4] Brain MRI Image Classification Using Probabilistic Neural Network and Tumor Detection Using Image Segmentation Prof. N.D.Pergad, Ms. Kshitija V.Shingare ISSN: $2278-1323$

[5] Brain Tumor Classification Using PNN And Clustering P.Sangeetha Department of Electronics and communication engineering, Nehru institute of Technology, Coimbatore,
Tamilnadu, India. Volume 3, Special Issue 3, March 20142014 International Conference on Innovations in Engineering and Technology (ICIET'14)

[6] Implementation of Classification System for Brain Cancer Using Backpropagation Network and MRI Prof. Vikas Gupta, Kaustubh S. Sagale Technocrats Institute of Technology, Bhopal, MP 978-14673-1719-1/12/\$31.00@2013IEEE

[7] D.F. Specht, "Probabilistic Neural Networks for Classification, mapping, or associative memory", Proceedings of IEEE International conference on neural networks, Vol. 1, IEEE press, New York, pp. 525-532, june 1988

[8] Brain Tumour classification, Detection and Segmentation Using Digital Image Processing and Probabilistic Neural Network Techniques Dr. P.V. RAMARAJU Department of Electronics and communication. SRKR ENGINEERING COLLEGE ANDHRA PRADESH, INDIA. SHAIK BAJI Department of Electronics and communication. SRKR ENGINEERING COLLEGE ANDHRA PRADESH, INDIA. Email: pvrraju50@gmail.com Email: baji .sk401@gmail.com. International Journal of Emerging Trends in Electrical and Electronics (IJETEE - ISSN: 2320-9569) Vol. 10, Issue. 10, Oct. 2014

[9] Feature Extraction and Texture Classification in MRI Jayashri Joshi1, Mrs.A.C.Phadke2 1. Marathwada Mitra Mandal's College of Engineering, Pune. 2. Maharashtra Institute of Technology, Pune 1. kjayashri@rediffmail.com 2. anu_phadke@yahoo.com Special Issue of IJCCT Vol. 2 Issue 2, 3, 4; 2010 for International Conference [ICCT-2010], 3rd-5th December 2010 